\title{
Left ventricular myocardial volume in CZT SPECT: Expanding the horizon?
}

\author{
Javier Gomez, MDa \\ a Division of Cardiology, John H. Stroger, Jr. Hospital of Cook County, Chicago, IL
}

Received Dec 3, 2019; accepted Dec 4, 2019

doi: 10.1007/s12350-019-01995-3

\section{See related article, pp. 1623-1633}

Myocardial perfusion imaging (MPI) with singlephoton emission computed tomography (SPECT) is a commonly used test in the evaluation of patients with suspected or known coronary artery disease due to its established diagnostic and prognostic value. ${ }^{1}$ Although traditionally, the presence and extent of myocardial perfusion defects have been considered the cornerstone in the diagnosis and risk stratification of patients, many additional non-perfusion variables have been shown to further improve the performance of cardiac SPECT imaging. Some of these variables include left ventricular (LV) ejection fraction, LV wall motion score, LV volume, ST segment changes, lung-heart ratio, transient ischemic dilation, and heart rate response. ${ }^{2}$ The advent of newer generation solid-state cameras equipped with cadmium-zinc-telluride (CZT) detectors has considerably improved spatial resolution and photon sensitivity compared to standard anger cameras. Such advances have allowed for a more precise three-dimensional reconstruction of the heart and thus more reliable measurements of LV myocardial volume (LVMV) and calculation of myocardial mass. Previous data have shown incremental prognostic value of LVMV and myocardial mass using different imaging modalities. ${ }^{3}$ In this regard, Gimelli et al., previously evaluated the feasibility of measuring LVMV using CZT SPECT cameras and demonstrated its high reproducibility and a good correlation with cardiac magnetic resonance derived values. ${ }^{4}$

Reprint requests: Javier Gomez, MD, Division of Cardiology, John H. Stroger, Jr. Hospital of Cook County, 1901 W. Harrison St., Suite \# 3620, Chicago, IL 60612, USA; jgomez@cookcountyhhs.org J Nucl Cardiol 2021;28:1634-5.

$1071-3581 / \$ 34.00$

Copyright (c) 2019 American Society of Nuclear Cardiology.
In this issue of the journal, Gimelli et al. evaluated the incremental diagnostic value of change in LVMV between rest and stress ( $\triangle \mathrm{LVMV}$ ) using CZT SPECT cameras and corridor 4DM-SPECT software (INVIAAnn Arbor, MI). ${ }^{5}$ The authors prospectively enrolled 512 patients with known or suspected CAD with high pretest probability ( $>65 \%$ based on Diamond and Forrester classification) who were referred for SPECT-MPI followed by invasive coronary angiography within 30 days regardless of SPECT-MPI results. The authors determined that LVMV measurement is expected to decline on the post-stress images. Using receiver operating characteristic curves, they identified a threshold of $\Delta \mathrm{LVMV} \leq 5 \mathrm{~mL}$ decrease between rest and stress (corresponding to $6 \%$ relative change) as having the best discriminatory capacity for detection of obstructive CAD. In a multivariate logistic regression model, $\Delta \mathrm{LVMV} \leq 5 \mathrm{~mL}$ was shown to be an independent predictor of obstructive CAD, and its use, in addition to MPI status (abnormal MPI defined as stress score of $\geq$ 4) was associated with improved patient classification using integrated discrimination and net reclassification improvement indices. ${ }^{6}$ The authors hypothesize that the phenomenon of reduction in LVMV at stress when compared to rest is related to increased inotropy and chronotropy during stress which in turn leads to compression of myocardial blood vessels and reduction in overall LVMV. In patients with CAD, this change is blunted by reduced inotropic and chronotropic augmentation leading to less pronounced reductions in LVMV. These results suggest that the measurement of $\triangle \mathrm{LVMV}$ using CZT SPECT cameras may improve the diagnostic accuracy of SPECT-MPI. Since the measurements are automatically and routinely generated, the additional time and effort involved to attain this data appear to be minimal. Furthermore, its use could prove valuable in cases of borderline or equivocal perfusion findings.

Despite the potential value of $\triangle \mathrm{LVMV}$ measurement, there are important factors that need to be 
considered carefully. The authors derived a value of LVMV decrease $\leq 5 \mathrm{~mL}$ as the optimal threshold to improve detection of obstructive CAD and then applied it in the same cohort. Deriving a diagnostic or prognostic marker and applying it in the same cohort without validation in a separate cohort is problematic. Any diagnostic threshold is expected to perform best in the cohort from which it was derived. Reproduction of the study findings and external validation of the proposed threshold are necessary before considering using the proposed variable clinically. The theoretical explanation proposed by the authors by which LVMV and mass decrease during stress is due to compression of intramyocardial blood vessels although this is not proven in humans. It is conceivable that the change in LVMV is the result of difference in partial volume averaging at the edges of the LV myocardial contours between rest and hyperdynamic stress, leading to a difference in the number of voxels being recruited for image reconstruction. Considering lack of external validation and unconfirmed mechanism of the study marker, the present study should be considered as hypothesis generating. Additional studies validating these results are required.

A limited number of patients with reduced ejection fraction or with significant left ventricular hypertrophy were included in the study. Thus, the generalizability of the study is limited, as it is unclear whether this parameter can be applied to patients in whom myocardial contractility lies at the extremes of the spectrum. Additionally, the prevalence of abnormal SPECT-MPI and obstructive CAD in the cohort studied was reported to be $43 \%$ and $53 \%$, respectively. These values are higher than those encountered in routine clinical practice. The authors explain this finding by the selected nature of the cohort studied (only patients with high pretest probability of CAD). It is unclear whether $\Delta \mathrm{LVMV} \leq 5 \mathrm{~mL}$ would have the same diagnostic value in an unselected cohort, especially in the current era of declining prevalence and severity of CAD. ${ }^{7}$ Finally, although $\triangle \mathrm{LVMV}$ may prove useful in cases of equivocal perfusion results, there is uncertainty regarding how should conflicting results be interpreted? Should patients with normal perfusion and abnormal $\triangle \mathrm{LVMV}$ be further investigated to detect or exclude CAD? Are patients with abnormal perfusion and normal $\triangle \mathrm{LVMV}$ at a lower risk? Are the findings reproducible using different SPECT image analysis software?

The authors should be commended for an interesting paper exploring the potential use of what may prove to be a novel parameter in the detection of CAD. However, additional research is needed to clarify the mechanism by which LVMV changes between rest and stress. Moreover, the independent and incremental diagnostic and prognostic value of $\triangle \mathrm{LVMV}$ needs to be validated in a different cohort of all comers for SPECTMPI. The potential role of $\triangle \mathrm{LVMV}$ in daily clinical practice needs to be better elucidated before it can be added to the battery of tools routinely used by clinicians for the diagnosis and management of patients with heart disease.

\section{References}

1. Shaw LJ, Iskandrian AE. Prognostic value of gated myocardial perfusion SPECT. J Nucl Cardiol 2004;11:171-85.

2. Bajaj NS, Singh S, Farag A, El-Hajj S, Heo J, Iskandrian AE, et al. The prognostic value of non-perfusion variables obtained during vasodilator stress myocardial perfusion imaging. J Nucl Cardiol 2016;23:390-413.

3. Stevens SM, Reinier K, Chugh SS. Increased left ventricular mass as a predictor of sudden cardiac death: Is it time to put it to the test? Circ Arrhythm Electrophysiol 2013;6:212-7.

4. Gimelli A, Liga R, Magro S, Novo S, Pedrinelli R, Petronio AS, et al. Evaluation of left ventricular mass on cadmium-zinc-telluride imaging: Validation against cardiac magnetic resonance. J Nucl Cardiol 2019;26:899-905.

5. Ficaro EP, Lee BC, Kritzman JN, Corbett JR. Corridor4DM: The Michigan method for quantitative nuclear cardiology. J Nucl Cardiol 2007; 14:455-65.

6. Gimelli A, Pugliese NR, Buechel RR, Bertasi M, Coceani M, Marzullo $\mathrm{P}$. Changes in left ventricle myocardial volume during stress test using cadmium-zinc-telluride cardiac imaging: Implications in coronary artery disease. J Nucl Cardiol 2019. https://doi. org/10.1007/s12350-019-01930-6.

7. Rozanski A, Gransar H, Hayes SW, Min J, Friedman JD, Thomson LE, et al. Temporal trends in the frequency of inducible myocardial ischemia during cardiac stress testing: 1991 to 2009. J Am Coll Cardiol 2013;61:1054-65.

Publisher's Note Springer Nature remains neutral with regard to jurisdictional claims in published maps and institutional affiliations. 